\title{
Effects of Nanosecond Repetitively Pulsed Plasma Discharges on a Propagating Hydrogen-Air Flame
}

\author{
Joshua A.T. Gray* and Deanna A. Lacoste ${ }^{\dagger}$ \\ Clean Combustion Research Center \\ King Abdullah University of Science and Technology (KAUST) \\ Thuwal, 23955-6900, Saudi Arabia
}

\begin{abstract}
Pressure-gain combustion cycles have been a topic of intense scientific research in recent years. Pulse detonation combustors are one promising technology for successfully implementing these pressure-gain cycles. These combustors frequently rely on obstacles for accelerating a propagating flame and inducing the deflagration-to-detonation transition. The use of obstacles, however, has several disadvantages. Replacing such obstacles with nanosecond repetitively pulsed plasma discharges may be advantageous to developing this technology further. Proof of concept has been achieved demonstrating the effectiveness of this strategy at enhancing the transition to detonation. This work is a closer investigation into the effect of these plasma discharges on flames with varying turbulence intensities and propagation velocities. For velocities below $200 \mathrm{~m} / \mathrm{s}$, the effect is minimal and transition to detonation does not occur. The effect of plasma discharges on flame acceleration also begins to diminish for the current configuration and pulse repetition frequency at speeds above $400 \mathrm{~m} / \mathrm{s}$. This give a range where nanosecond repetitively pulsed plasma discharges may be used effectively for efficient flame acceleration and transition to detonation.
\end{abstract}

\section{Introduction}

$\mathrm{D}$ UE to the inherent efficiency gains associated with pressure-gain combustion, much research effort has been dedicated to this field in recent years [1--3]. Although much attention is currently being given to the rotating detonation engine, other concepts, such as the pulse jet and the pulse detonation engine continue to be viable concepts for advanced pressure-gain cycles. One challenge with the pulse detonation combustor (PDC) is the reliable and efficient generation of the deflagration-to-detonation transition (DDT). This is typically achieved using varying numbers of obstacles of various geometries [4-6]. In this way, the flame is accelerated by flame folding and the associated increase in the turbulent flame speed followed by DDT resulting frequently from shock-obstacle interaction. However, these obstacles are subjected to intense thermal loading and subsequent failure. Furthermore, the presence of obstacles in the detonation tube has a direct impact on the efficiency of the engine, due to the associated pressure drop and heat losses [7]. Arc discharges have been successfully used to accelerate propagating flames and enhance the occurrence of DDT [8, 9]. However, the energy deposition of these discharges is very large (on the order of $100 \mathrm{~J}$ ). The authors have proposed a new concept of replacing physical obstacles with nanosecond repetitively pulsed (NRP) plasma discharges [10]. Indeed, NRP discharges have shown much promise in enhancing the chemical kinetics in plasma-assisted combustion applications in order to improve ignition, control combustion instabilities, and extend lean blow-off limits [11, 12]. The improved ignition aspect of these discharges has already been applied to pulse detonation combustors to reduce the run-up time to DDT [13-16].

In a recent study by the authors, proof of concept has been demonstrated using NRP plasma discharges on a propagating flame to significantly increase flame acceleration and decrease the run-up length to DDT [10]. This strategy has proven to be very robust and reproducible. The propagation velocity of the reaction front immediately after the discharge location is seen to more than double when compared to cases in which no plasma forcing has been applied. The setup involved using a single orifice plate obstacle followed by a pin-ring electrode configuration, between which the NRP discharges are applied. Additionally, the energy deposition was quantified for both the initial ignition spark as well as for the NRP discharges. It has been determined that a combination of an ignition spark with an energy deposition of $9 \mathrm{~mJ}$ and a series of NRP discharges with a total energy deposition of $35 \mathrm{~mJ}$ results in consistent DDT, while a more powerful ignition spark with an energy deposition of $70 \mathrm{~mJ}$ does not result in DDT. Thus, using NRP discharges to

\footnotetext{
*Postdoctoral Fellow, Clean Combustion Research Center, 4700 KAUST, Thuwal, 23955-6900, Saudi Arabia, AIAA Member

${ }^{\dagger}$ Assistant Professor, Clean Combustion Research Center, 4700 KAUST, Thuwal, 23955-6900, Saudi Arabia
} 
accelerate a propagating flame is an energetically more efficient strategy. In this context, the objective of the present study is to investigate how the flame propagation speed in the vicinity of the electrodes influences the effectiveness of this strategy.

\section{Experimental Setup and Procedure}

A schematic of the experimental setup is detailed in Fig. 1. The detonation chamber consists of six stainless steel tube sections, each with an inner diameter of $39 \mathrm{~mm}$ and a length of $540 \mathrm{~mm}$. A standard automotive spark plug is installed in the head wall of the detonation chamber for ignition of the combustible mixture. A quartz glass tube is installed at $1080 \mathrm{~mm}$ (after the second tube section). The quartz glass tube serves to electrically insulate the electrodes for the NRP plasma from the test bench as well as to provide optical access. After the electrodes, the four remaining tube sections are installed, resulting in a total length of the test bench of $3350 \mathrm{~mm}$. Two ionization probes (standard automotive spark plugs) are installed in each tube section, as shown in Fig. 1. This results in measurement positions at eight axial locations beginning at $1315 \mathrm{~mm}$ with a separation distance of $270 \mathrm{~mm}$. The measurement signals are acquired with a high-speed data acquisition system (NI, PXI-5105 Oscilloscope) at $30 \mathrm{MHz}$.

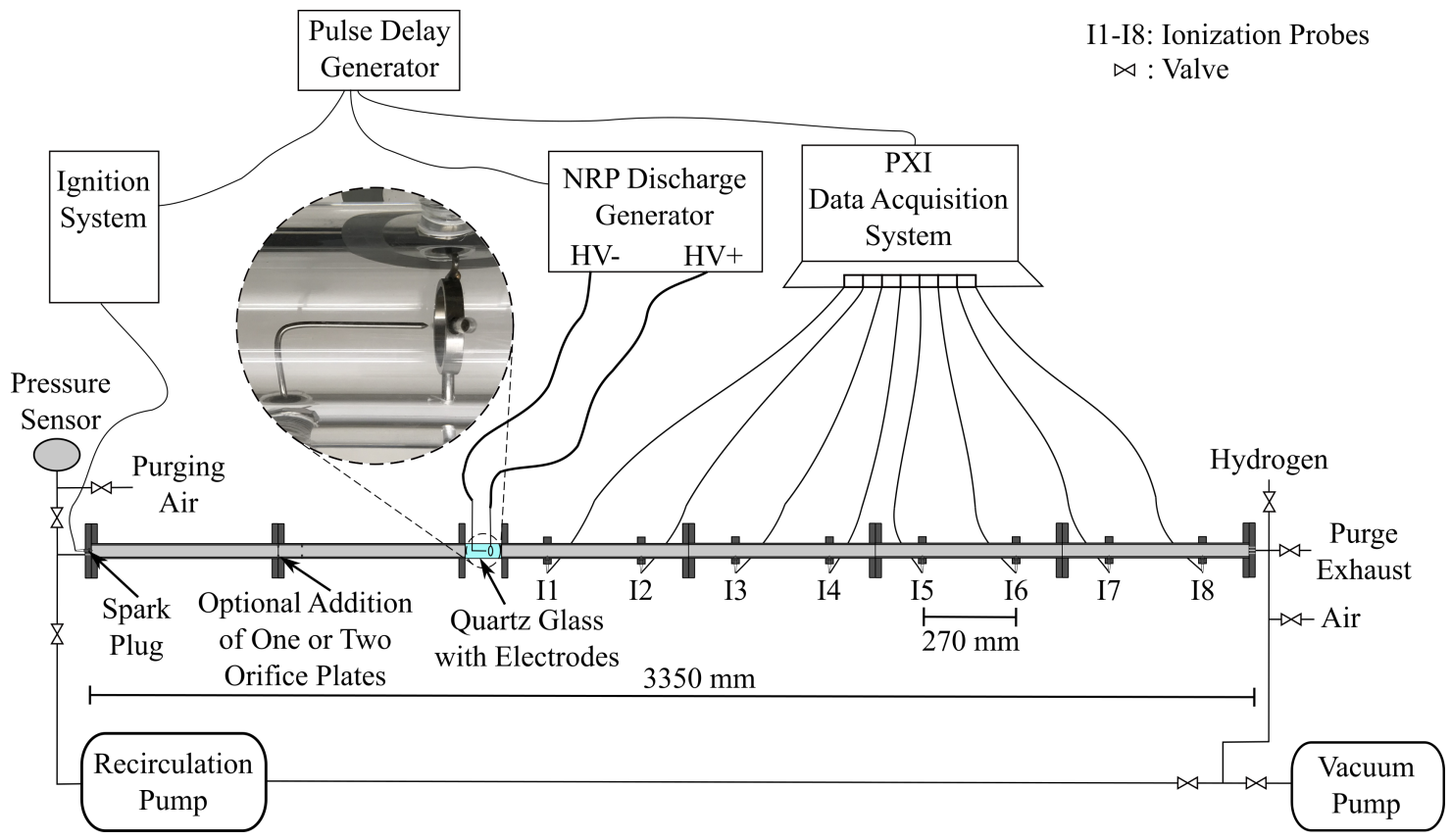

Fig. 1 Schematic of experimental test bench, including a detailed image of the electrode configuration.

The electrodes are constructed in a pin-ring configuration and are made of stainless steel. The cathode is a sharpened pin with a diameter of $1.6 \mathrm{~mm}$. The tip of the cathode is positioned $5 \mathrm{~mm}$ upstream of the ring electrode, which is positioned at $1150 \mathrm{~mm}$ from the head wall. The ring electrode has an inner diameter of $20 \mathrm{~mm}$, an outer diameter of $24 \mathrm{~mm}$, and a thickness of $4 \mathrm{~mm}$. It is held in place by three stainless steel dowel pins, which are inserted through the wall of the quartz glass tube. The NRP plasma discharges are obtained by applying high-voltage pulses with a width of around $15 \mathrm{~ns}$ and an amplitude of $24.5 \mathrm{kV}$ to the electrodes. These pulses are produced by an NRP discharge generator (FID FPD 25-100MC2), which provides a positive high-voltage pulse of $12.25 \mathrm{kV}$ and a negative high-voltage pulse of $-12.25 \mathrm{kV}$. The pulse repetition frequency is held constant at $100 \mathrm{kHz}$.

The test bench is purged for ten minutes with pressurized air in order to expel any remaining water vapor from the previous test runs, which even in small quantities has shown to have a significant effect on the repeatability of the experiments. Then, the test stand is evacuated to 10 mbar with a vacuum pump (Edwards RV8) and filled to a pressure of 1 bar with a stoichiometric hydrogen-air mixture using the partial pressures method with the help of a static pressure gauge (Keller Leo 3). The hydrogen and air are then mixed within the test stand for three minutes using a recirculation pump (KNF Laboport N 842.3 FT.18). Subsequently, the mixture is allowed to settle for one minute before it is ignited by the spark plug in the head wall using a $1 \mathrm{~ms}$ pulse from a high-voltage power amplifier (Trek PD07016) with an 
amplitude of $6 \mathrm{kV}$. The propagation velocity of the reaction front is determined using the time-of-flight method from the signals of the ionization probes. Additionally, high-speed $\mathrm{OH}$-chemiluminescence imagery is conducted at $50 \mathrm{kHz}$ for cases without plasma actuation using a CMOS camera (LaVision HSS8) coupled with an intensifier (LaVision IRO). This imagery is done to gain an understanding of the flame propagation in the inter-electrode area as well as to characterize the effect of the electrodes themselves on the flame propagation. The ignition spark, measurement acquisition system, high-speed camera, and NRP plasma discharges are synchronized using a pulse/delay generator (Berkley Nucleonics Corporation, Model 575).

In order to vary the turbulent properties of the flame at the position of the electrodes, various numbers of orifice plates are installed at the position indicated in Fig. 1 $1540 \mathrm{~mm}$ from the headwall) by adding an insert between the flanges. Either zero, one, or two orifice plates are installed. The blockage ratio is 0.43 . For the case of two orifice plates, the second orifice plate is attached to the insert by means of four $2 \mathrm{~mm}$ threaded rods with a separation distance of $85 \mathrm{~mm}$. This blockage ratio and separation distance has been shown to be optimal for this mixture and tube diameter ([4, 6, 17]). Finally, control investigations are conducted with one orifice plate at $540 \mathrm{~mm}$ and one orifice plate with the same blockage ratio installed in the quartz tube at the position of the ring electrode.

\section{Results}

The high-speed images allow for the flame propagation speed to be determined in the vicinity of the electrodes. Sample images are shown in Fig. 2 The increase in intensity of the flames is immediately evident as more orifice plates are introduced upstream of the visualization area. This is a direct consequence of an increase in the turbulent burning rate and is also reflected in the increase in propagation velocity of the flames. The ring-electrode is visible in Fig. 2 $\mathrm{b}$, although the field of view is slightly shifted for the cases using orifice plates in order to capture more of the flame propagation downstream of the electrodes. Furthermore, the cases with zero or one orifice plate exhibit "finger-like" flames, with unburnt zones along the wall. The flame shape is due to the boundary layer effects. As the propagation velocity and turbulence increase, the flame becomes flatter and the reaction propagates more quickly into the boundary layer.

The propagation speed along the tube can be determined by measuring the flame displacement between two consecutive images. These are shown in Fig. 3 for the three configurations. The data from ten test runs each for the cases with one and two orifice plates and five test runs for the case with no orifice plate is shown. Due to the lower propagation speed of the latter case, more data points can be obtained from each test run. The uncertainty in determining the forward-most flame position is $\pm 0.25 \mathrm{~mm}$, resulting in an uncertainty in the propagation speed obtained from two images of $\pm 25 \mathrm{~m} / \mathrm{s}$. The case with no orifice plate shows no significant acceleration along the tube with an average propagation speed of around $120 \mathrm{~m} / \mathrm{s}$ before the electrodes. After the flame passes the electrodes, a modest acceleration of between 10-20\% is observed, although this is within the uncertainty of the measurements. With one orifice plate installed, the flame tends to accelerate before the electrodes with an average speed of around $250 \mathrm{~m} / \mathrm{s}$ increasing to an average speed of $350 \mathrm{~m} / \mathrm{s}$. After passing the electrodes, the average speed increases to around $400 \mathrm{~m} / \mathrm{s}$, comparable to the previous case (10-20\%). With two orifice plates installed, the flame accelerates from around $350 \mathrm{~m} / \mathrm{s}$ to $450 \mathrm{~m} / \mathrm{s}$ before the electrodes. However, interaction with the ring electrode is more pronounced with a sharp increase in the average propagation speed to between $600 \mathrm{~m} / \mathrm{s}$ and $700 \mathrm{~m} / \mathrm{s}$. This is an increase of 30-50\%. Although the blockage ratio of the ring electrode is small $(0.122)$ and its effect on the flame propagation speed at lower velocities is minimal, its mere presence at higher velocities has a significant effect on the flame propagation.

Using the high-speed imagery, an optimum delay is determined between the ignition and the beginning of the train of NRP plasma pulses. This is necessary due to the inherent stochastic nature of turbulent flame propagation. A series of 100 pulses is used and the delay is chosen so that the 75th pulse is applied at a time corresponding to the average arrival time of the flame at the inter-electrode area. A summary of the average arrival times for the flames in the inter-electrode area for the various configurations and the corresponding delay between the ignition and the beginning of plasma actuation is given in Table 1 .

The investigations with one orifice plate serve to confirm the results reported in [10]. The flame propagation velocities with and without plasma actuation are presented in Fig. 4. alongside the propagation velocities obtained with an orifice plate installed at the position of the ring electrode. For each configuration, 10 test cases are presented. For the control case with a second orifice installed instead of the electrodes (blue squares), there are two characteristic propagation modes. In 50\% of the test runs, DDT occurs almost immediately after the flame interacts with the second orifice plate. However, in the other test runs, DDT occurs only just before $2.25 \mathrm{~m}$ or $2.5 \mathrm{~m}$. Contrasting this to the case, where the orifice is again removed and replaced by the electrodes reveals a significantly different behavior. Two 
No Orifice

a)
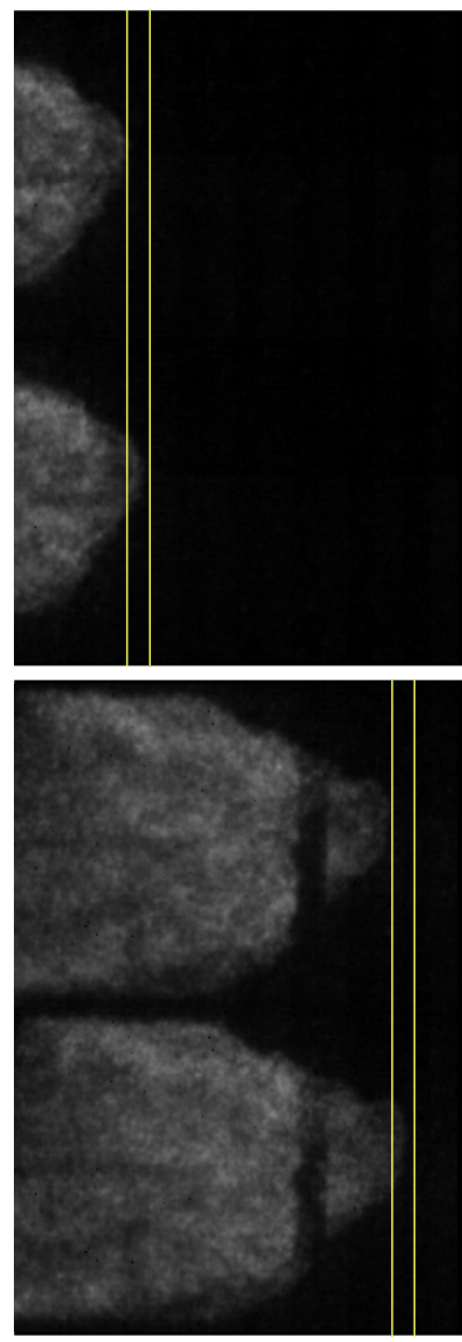

One Orifice
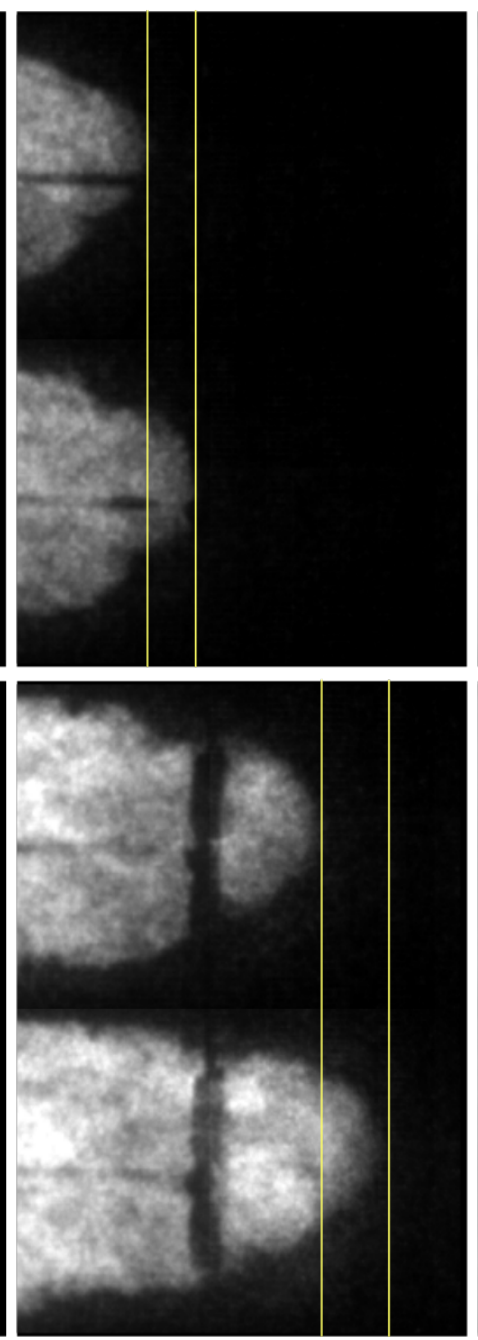

Two Orifices
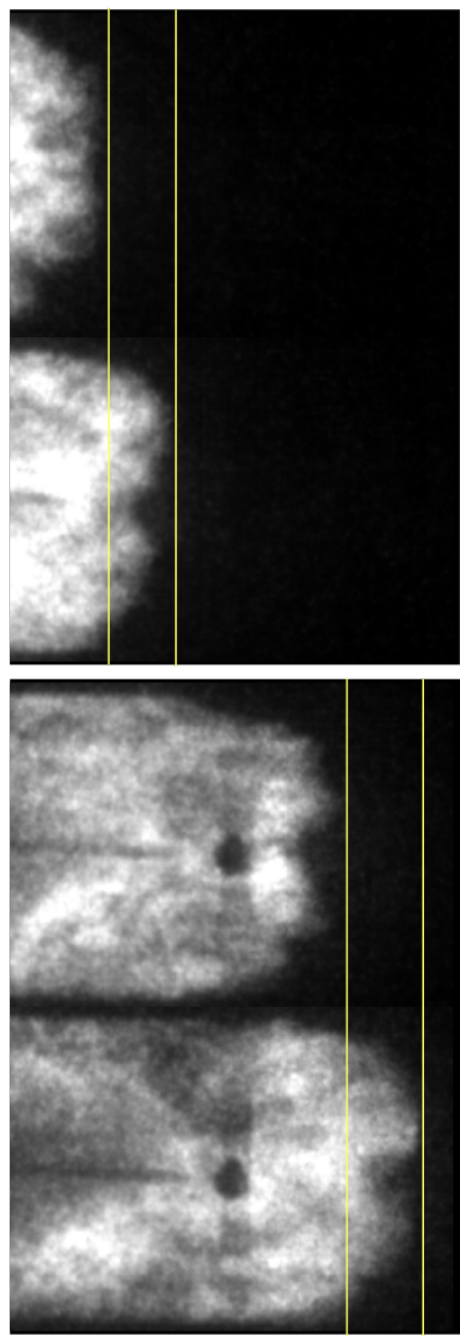

Fig. 2 Images of propagating hydrogen-air flames in three configurations: with no orifice plate, one orifice plate, and two orifice plates upstream of the visualization section. Images are shown at two positions: a) upstream of the electrodes and b) downstream of the electrodes. Lines are draw to show the forward-most point in the flame front. The time step between each pair of images is $20 \mu \mathrm{s}$.

propagation modes are also seen here as previously reported. In two cases, extreme deceleration of the flame is observed due to the "tulip flame" phenomenon [18], a type of Rayleigh-Taylor instability, and subsequent coupling with the acoustic modes of the tube [19, 20]. In the remaining cases, the flame does not exhibit said deceleration and continues to moderately accelerate. However, without plasma actuation, DDT does not occur within the length of the tube. When NRP plasma is applied, the flame is accelerated significantly, albeit not to the same extent as with the second orifice plate. Notice the absence of measurements for this case at $1.7 \mathrm{~m}$. This is due to the electromagnetic noise caused by the pulsing, preventing this point from being captured. Before $2.5 \mathrm{~m}, 40 \%$ of the cases transition to detonation. By $2.8 \mathrm{~m}$ detonation has occurred for all cases. However, in one case, the detonation decouples and decelerates before the last ionization probe. In fact, this behavior is also seen upstream at around $2 \mathrm{~m}$ from the headwall for five cases. This could be due to a local explosion ahead of the flame that does not transition to a detonation, but still registers as a reaction front at the ionization probe. Nevertheless, these measurements validate the previous proof of concept reported in [10] that NRP plasma discharges can successfully accelerate the transition to detonation for flames propagating at the velocities exhibited in this configuration. 


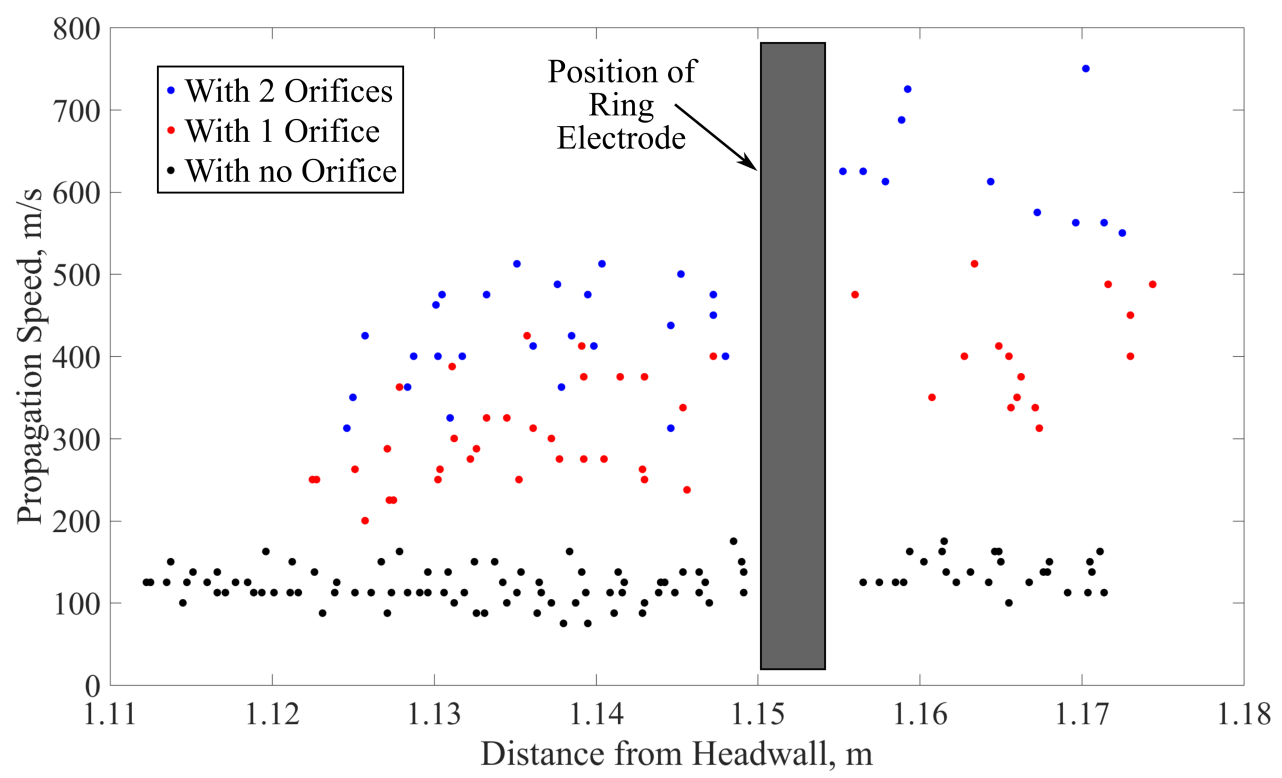

Fig. 3 Flame propagation velocities in the quartz glass tube obtained from flame displacement in the high-speed images. The velocities are shown for three configurations: no orifice plate (black), one orifice plate (red), and two orifice plates (blue).

Table 1 Average arrival times of the propagating flames in each configuration and the corresponding delay applied between ignition and application of the first NRP plasma pulse.

\begin{tabular}{ccc}
\hline Configuration & Average Arrival Time, ms & Applied Delay, ms \\
\hline No Orifice & 18.77 & 18.02 \\
\hline One Orifice & 12.19 & 11.44 \\
\hline Two Orifices & 11.55 & 10.80 \\
\hline
\end{tabular}

Applying the same discharges with the configuration without an orifice plate proves less effective. These measurements are shown in Fig. 5. As can be seen, without an orifice plate, the flame does not accelerate along the length of the tube. In fact, the flame tends to decelerate and propagate at differing speeds before reaching the end of the tube. This again indicates the presence of the Rayleigh-Taylor instability and oscillations in the propagation speeds. Even using NRP discharges, no effect on the flame acceleration is evident and the flame does not exceed $150 \mathrm{~m} / \mathrm{s}$. This is consistent with the propagation speeds obtained from the high-speed images, in which the flame was consistently between $100 \mathrm{~m} / \mathrm{s}$ and $150 \mathrm{~m} / \mathrm{s}$ near the inter-electrode area.

Adding two orifices upstream of the electrodes, once again, proved more effective at accelerating the flame. However, the effect was not as pronounced as for the configuration with one orifice. These measurements are presented in Fig.6 For these measurements, the number of pulses was reduced from 100 to 85 in order to prevent the electromagnetic noise from interfering with the ionization probe signals. Even with this reduction, however, the ionization probe at position I2 (see Fig. 11) was compromised, resulting in one less measurement point. Because the influence of the plasma discharges on the flame propagation is not immediately evident in the figure, the percentage of the transition at each probe position is given in Table 2. Here it is clear that the flame acceleration is enhanced by the NRP pulses, even resulting in very early DDT in $20 \%$ of the cases. However, the effect is not as pronounced as in the case with one orifice plate. This may be due to the limited number of discharges that interact with the flame. At a propagation speed of $450 \mathrm{~m} / \mathrm{s}$ at the inter-electrode area and a pulse repetition frequency of $100 \mathrm{kHz}$, only about two discharges occur within $10 \mathrm{~mm}$ ahead of the flame. Additionally, the increased fluid-dynamic effect merely due to the presence of the ring electrode may play 
a role, even without the application of NRP discharges.

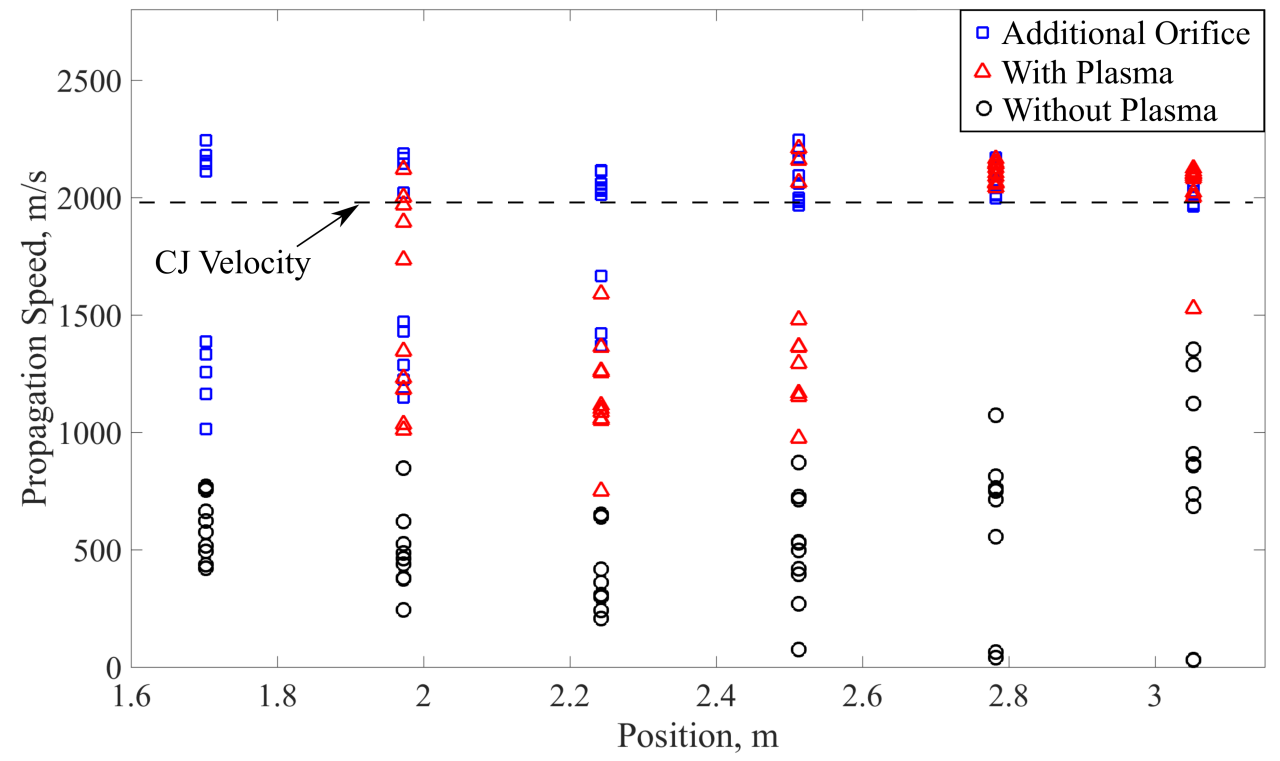

Fig. 4 Propagation speeds obtained from time-of-flight measurements using the ionization probes for the case with one orifice plate installed upstream of the electrodes. Ten test runs each are presented for tests with NRP plasma discharge and without.

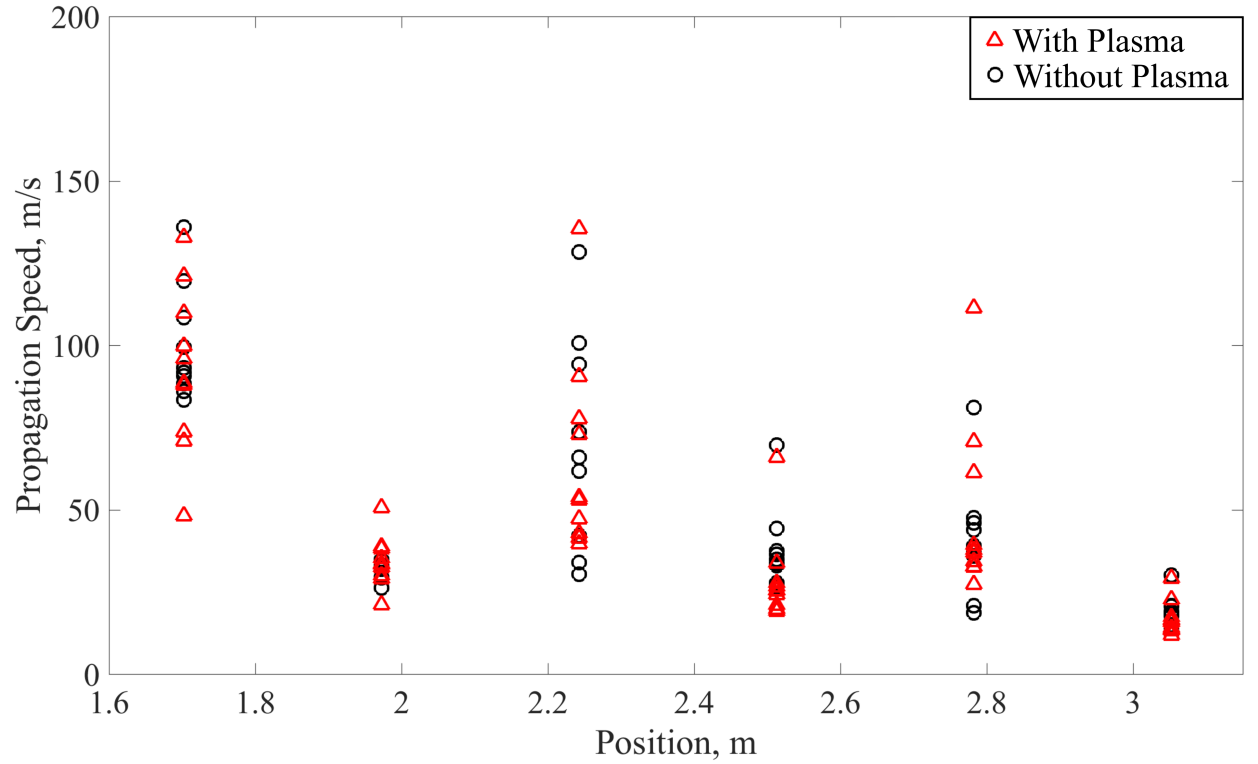

Fig. 5 Propagation speeds obtained from time-of-flight measurements using the ionization probes for the case with no orifice plate installed upstream of the electrodes. Ten test runs each are presented for tests with NRP plasma discharge and without. 


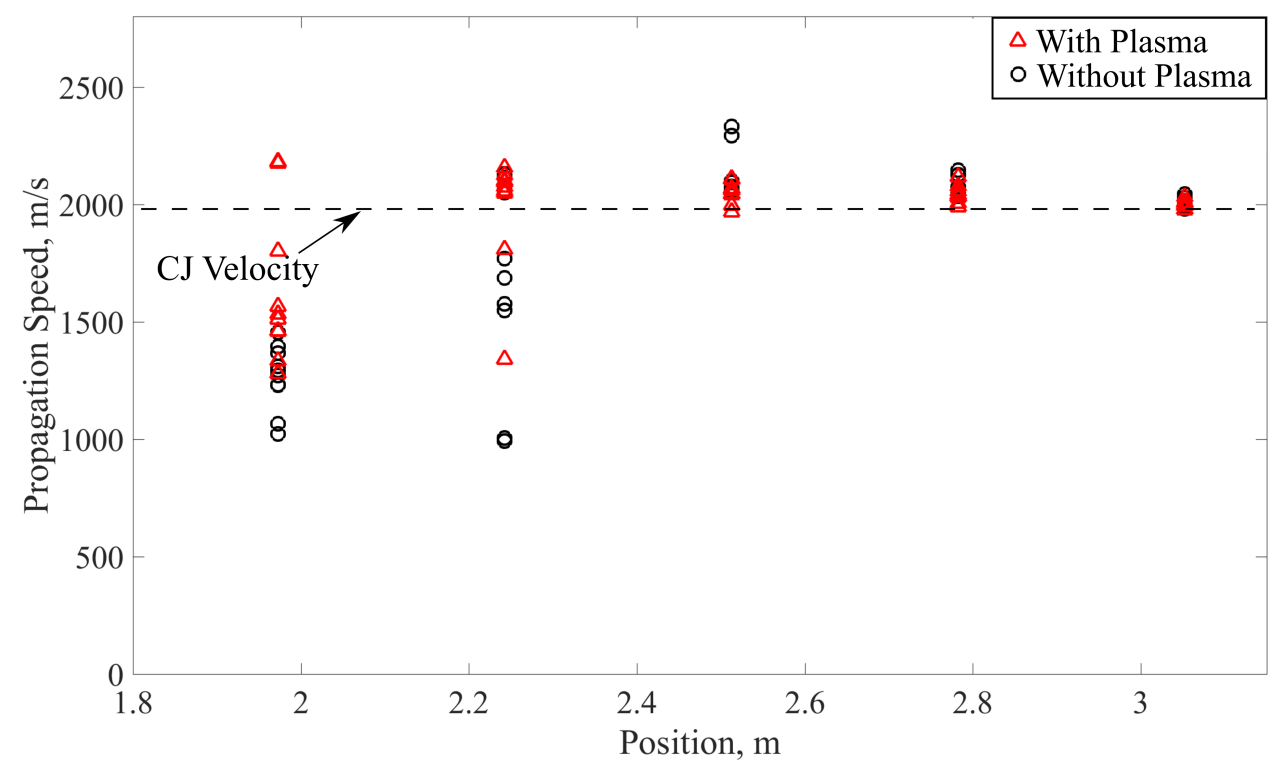

Fig. 6 Propagation speeds obtained from time-of-flight measurements using the ionization probes for the case with two orifice plates installed upstream of the electrodes. Ten test runs each are presented for tests with NRP plasma discharge and without.

Table 2 Percentage of successful transition to detonation with respect to the ionization sensors for cases with and without plasma actuation for the configuration with two orifice plates installed upstream of the electrodes.

\begin{tabular}{cccccc}
\hline & DDT before I3 & DDT before I4 & DDT before I5 & DDT before I6 & DDT before I7 \\
\hline Without NRP Plasma & $0 \%$ & $40 \%$ & $100 \%$ & $100 \%$ & $100 \%$ \\
\hline With NRP Plasma & $20 \%$ & $80 \%$ & $100 \%$ & $100 \%$ & $100 \%$ \\
\hline
\end{tabular}

\section{Conclusions}

In this work, the effect of NRP plasma discharges on propagating hydrogen-air flames has been evaluated for flames at various speeds. The speeds have been controlled by varying the number of orifice plates installed upstream of the inter-electrode area. The effect of the physical presence of the electrodes in the tube on the flame acceleration without plasma actuation has been quantified and shown to be minimal for speeds below $400 \mathrm{~m} / \mathrm{s}$, beyond which it may accelerate the flame by as much as 50\%. The case with one orifice plate serves to confirm previously published data from the authors and shows that NRP plasma discharges have a significant effect on flame acceleration and subsequent DDT in this regime. At lower velocities (with no orifice plate), the plasma discharges have no discernible effect on flame acceleration. At higher velocities (with two orifice plates), the effect also becomes less pronounced. Therefore, the range of propagation velocities for which the NRP plasma discharges at $100 \mathrm{kHz}$ with a pin-ring configuration are efficient is between about $200 \mathrm{~m} / \mathrm{s}$ and $400 \mathrm{~m} / \mathrm{s}$. At higher speeds, increasing the pulse repetition rate or changing the electrode configuration may improve performance.

\section{References}

[1] Paxson, D., and Kaemming, T., "Influence of Unsteadiness on the Analysis of Pressure Gain Combustion Devices," Journal of Propulsion and Power, Vol. 30 (2), 2014, pp. 377-383.

[2] Frolov, S., Aksenov, V., and Ivanov, V., "Experimental proof of Zel'dovich cycle efficiency gain over cycle with constant pressure combustion for hydrogen-oxygen fuel mixture," International Journal of Hydrogen Energy, Vol. 40(21), 2015, pp. $6970-6975$. 
[3] Lisanti, J., and Roberts, W., Pulse Combustor Driven Pressure Gain Combustion for High Efficiency Gas Turbine Engines. In. Agarwal A., De S., Pandey A., Singh A. (eds). Combustion for Power Generation and Transportation., Springer, Singapore, 2017.

[4] Lee, J., Knystautas, R., and Chan, C., “Turbulent flame propagation in obstacle-filled tubes,” Symposium (International) on Combustion, Vol. 20, 1985, pp. 1663-1672.

[5] Schauer, F., Stutrud, J., and Bradley, R., "Detonation initiation studies and performance results for pulsed detonation engine applications," 39th Aerospace Sciences Meeting and Exhibit, 2001.

[6] Ciccarreli, G., and Dorofeev, S., "Flame Accleration and Transition to Detonation in Ducts," Progress in Energy and Combustion Science, Vol. 34(4), 2008, pp. 499-550.

[7] Paxson, D., and Kaemming, T., "Performance Impact of Deflagration to Detonation Transition Enhancing Obstacles," 37th Aerospace Sciences Meeting and Exhibit including The New Horizons Forum and Aerospace Exposition, 2009.

[8] Frolov, S., Basevich, V., Aksenov, V., and Polikhov, S., "Detonation initiation by controlled triggering of electric discharges," Journal of Propulsion and Power, Vol. 19(4), 2003, pp. 573-580.

[9] Frolov, S., Basevich, V., Aksenov, V., and Polikhov, S., "Optimization study of spray detonation initiation by electric discharges," Shock Waves, Vol. 14(3), 2005, pp. 175-186.

[10] Gray, J., and Lacoste, D., "Enhancement of the Transition to Detonation of a Turbulent Hydrogen-Air Flame by Nanosecond Repetitively Pulsed Plasma Discharges," Combustion and Flame, Vol. 199, 2019, pp. 258-266.

[11] Starikovskaia, S., "Plasma assisted ignition and combustion," Journal of Physics D: Applied Physics, Vol. 39, 2006, pp. R265-R299.

[12] Ju, Y., and Sun, W., "Plasma assisted combustion: Dynamics and chemistry," Progress in Energy and Combustion Science, Vol. 48, 2015, pp. 21-83.

[13] Zhukov, V., and Starikovskii, A., "Effect of a nanosecond gas discharge on deflagration to detonation transition," Combustion Explosion and Shock Waves, Vol. 42 (2), 2006, pp. 195-204.

[14] Busby, K., Corrigan, J., Yu, S., Williams, S., Carter, C., Schauer, F., Hoke, J., Cathey, C., and Gundersen, M., "Effects of corona, spark and surface discharges on ignition delay and deflagration-to-detonation times in pulsed detonation engines," $45 \mathrm{th}$ AIAA Aerospace Sciences Meeting and Exhibit, 2007.

[15] Rakitin, A., and Starikovskii, A., "Mechanisms of deflagration-to-detonation transition under initiation by high-voltage nanosecond discharges," Combustion and Flame, Vol. 155 (1-2), 2008, pp. 343-355.

[16] Starikovskiy, A., Aleksandrov, N., and Rakitin, A., "Plasma-assisted ignition and deflagration-to-detonation transition," Phil. Trans. R. Soc. A, Vol. 370, 2012, pp. 740-773.

[17] Gray, J., Paschereit, C., and Moeck, J., "An Experimental Study of Different Obstacle Types for Flame Acceleration and DDT," In: King R. (eds) Active Flow and Combustion Control 2014. Notes on Numerical Fluid Mechanics and Multidisciplinary Design. Springer, Cham, Vol. 127, 2015, pp. 265-279.

[18] Clanet, C., and Searby, G., “On the "Tulip Flame” Phenomenon,” Combustion and Flame, Vol. 105, 1996, pp. $225-238$.

[19] Kerampran, S., Desbordes, D., and Veyssiere, B., "Study of the mechanism of flame acceleration in a tube of constant cross section," Combustion Science and Technology, Vol. 158, 2000, pp. 71-91.

[20] Gray, J., Moeck, J., and Paschereit, C., "Flame Propagation from the Closed End of an Open-Ended Tube: An Analysis of the Effects of Fuel Type, Tube Length, and Equivalence Ratio and an Insight into Flame Dynamics," 49th AIAA/ASME/SAE/ASEE Joint Propulsion Conference, 2013. 